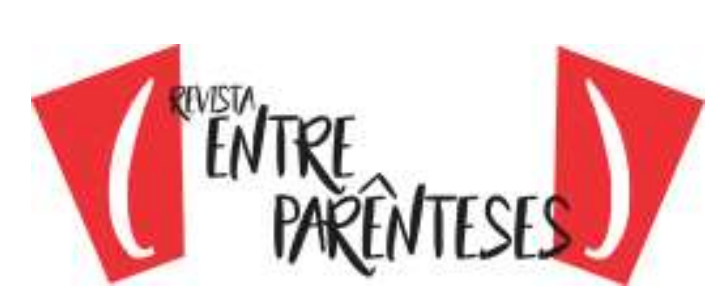

ISSN 2238-4502

\title{
LEVANTAMENTO SOBRE HÁBITOS DE LEITURA E ESCRITA ENTRE ESTUDANTES DE ENGENHARIA
}

\author{
Rafaela Giani de Resende \\ Universidade Federal do Triângulo Mineiro \\ (rafaelagiani@hotmail.com) \\ José Thiago da Rocha Neto \\ Universidade Federal do Triângulo Mineiro
}

\author{
Sofia Pelegrini Tristão \\ Universidade Federal do Triângulo Mineiro
}

\author{
Beatriz Gaydeczka \\ Universidade Federal do Triangulo \\ Mineiro/FAPEMIG \\ (beatriz.gaydeczka@uftm.edu.br)
}

\begin{abstract}
Resumo
Este artigo, produto da experiência de uma sequência didática para aprendizagem de gêneros acadêmicos, visa a apresentar dados de um levantamento sobre os hábitos de leitura e escrita entre estudantes de engenharia. Foi aplicado um instrumento online (Google Forms) com cinco questões (abertas e fechadas) sobre o tema. Verificou-se que grande parte dos estudantes possuem o hábito de leitura, sendo que a maioria se dedica a ler artigos e livros acadêmicos. A respeito do hábito de escrita, observou-se que ocorre em frequência menor e está vinculada às necessidades do curso, destacando-se como maiores dificuldades: a) transferir as ideias para o papel; b) organizar as ideias de forma coerente. De maneira geral, a existência de disciplinas que enfoquem escrita específica para estudantes de engenharia, juntamente com o esforço do próprio aluno, auxiliaria utilização de técnicas para desenvolver a escrita tanto pessoal quanto profissionalmente, como produção de textos técnicos, manuais de instrução ou normas operacionais.
\end{abstract}

Palavras-chave: Competências. Habilidade de comunicação. Gêneros textuais/discursivos.

DOI: https://doi.org/10.32988/rep.v10n1.1118

Dossiê "Possibilidades de trabalho com a Língua Portuguesa"

\begin{tabular}{|c|c|c|c|c|c|}
\hline Revista (Entre Parênteses) & Alfenas, MG & v. 10 & n. 1 & $1-20$ & $\mathrm{e} 021002$ \\
\hline $\begin{array}{r}\text { Depart } \\
\text { Rua Gabriel } \\
\text { https://publicaco }\end{array}$ & $\begin{array}{l}\text { Universid } \\
\text { nto de Letras - } \\
\text { teiro da Silva, } 7 \\
\text { nifal-mg.edu.l }\end{array}$ & $\begin{array}{l}\text { ederal } \\
\text { uto de } \\
\text { Alfenas } \\
\text { evistas }\end{array}$ & $\begin{array}{l}\text { nas } \\
\text { is Hu } \\
\text { CEP } \\
\text { x.php }\end{array}$ & $\begin{array}{l}\text { e Letr } \\
1-001 \\
\text { epare }\end{array}$ & asil \\
\hline
\end{tabular}




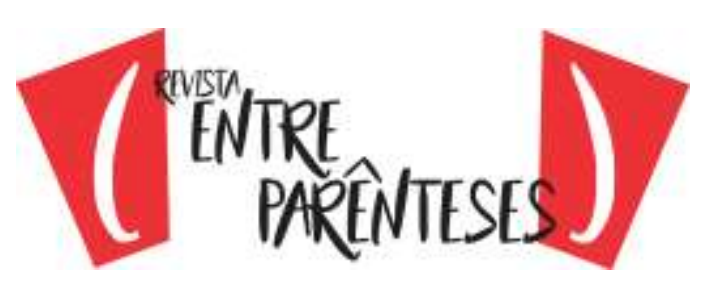

ISSN 2238-4502

\section{Rafaela Giani de Resende}

Graduada em Engenharia Elétrica da Universidade Federal do Triângulo Mineiro (UFTM, 2020), participou de vários projetos de iniciação científica, de extensão e de monitoria. Estagiou e foi efetivada na Procter and Gamble como gerente de vendas.

Estudante de Graduação de Engenharia Elétrica da Universidade Federal do Triângulo Mineiro (UFTM).

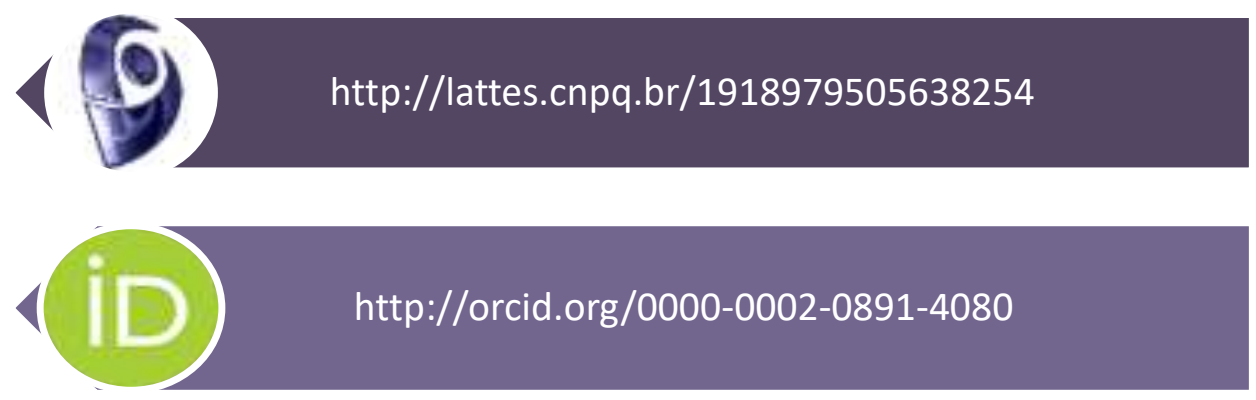

DOI: https://doi.org/10.32988/rep.v10n1.1118

Dossiê "Possibilidades de trabalho com a Língua Portuguesa"

v. 10

n. 1

$1-20$

$\mathrm{e} 021002$




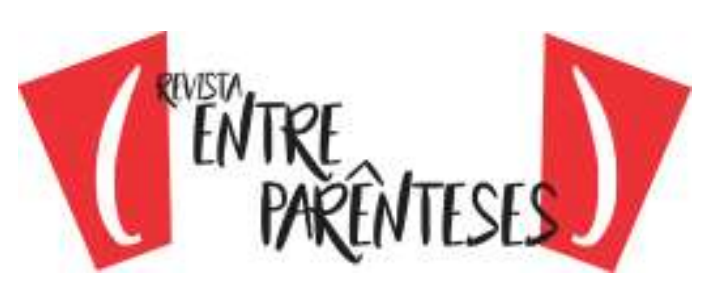

ISSN 2238-4502

\section{Sofia Pelegrini Tristão}

Estudante de Graduação em Engenharia Química da Universidade Federal do Triângulo Mineiro (UFTM).

\section{http://lattes.cnpq.br/7944883413085406}

http://orcid.org/0000-0002-2868-9802

\section{José Tiago da Rocha Neto}

Estudante de Graduação em Engenharia Mecânica da Universidade Federal do Triângulo Mineiro (UFTM).

\section{http://lattes.cnpq.br/2514907164354784}

\section{http://orcid.org/0000-0002-2668-7539}

DOI: https://doi.org/10.32988/rep.v10n1.1118

Dossiê "Possibilidades de trabalho com a Língua Portuguesa"

v. 10

n. 1

$1-20$

e021002




\section{Beatriz Gaydeczka}

Graduada em Pedagogia pela Universidade do Contestado (UnC, 2003), em Licenciada em Letras pela Universidade Estadual do Paraná (UNESPAR, 2003), mestrado em Linguística Aplicada pela Universidade de Taubaté (UNITAU, 2006) e doutorado em Letras na Universidade de São Paulo (USP, 2012). É Professora Associada na Universidade Federal do Triângulo Mineiro - UFTM, no Instituto de Ciências Tecnológicas e Exatas (ICTE). Atua na Graduação e na Pós-Graduação (Programa de Mestrado Profissional em Inovação Tecnológica) enfocando

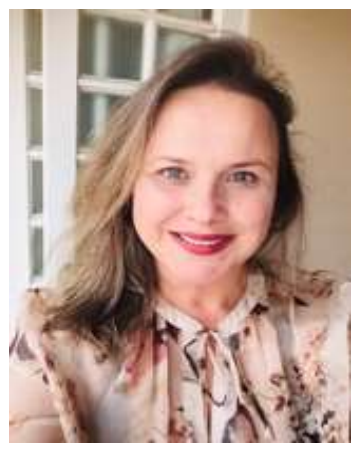
metodologia científica, comunicação, leitura e produção de textos técnicos para as engenharias, discurso acadêmico e propriedade intelectual.

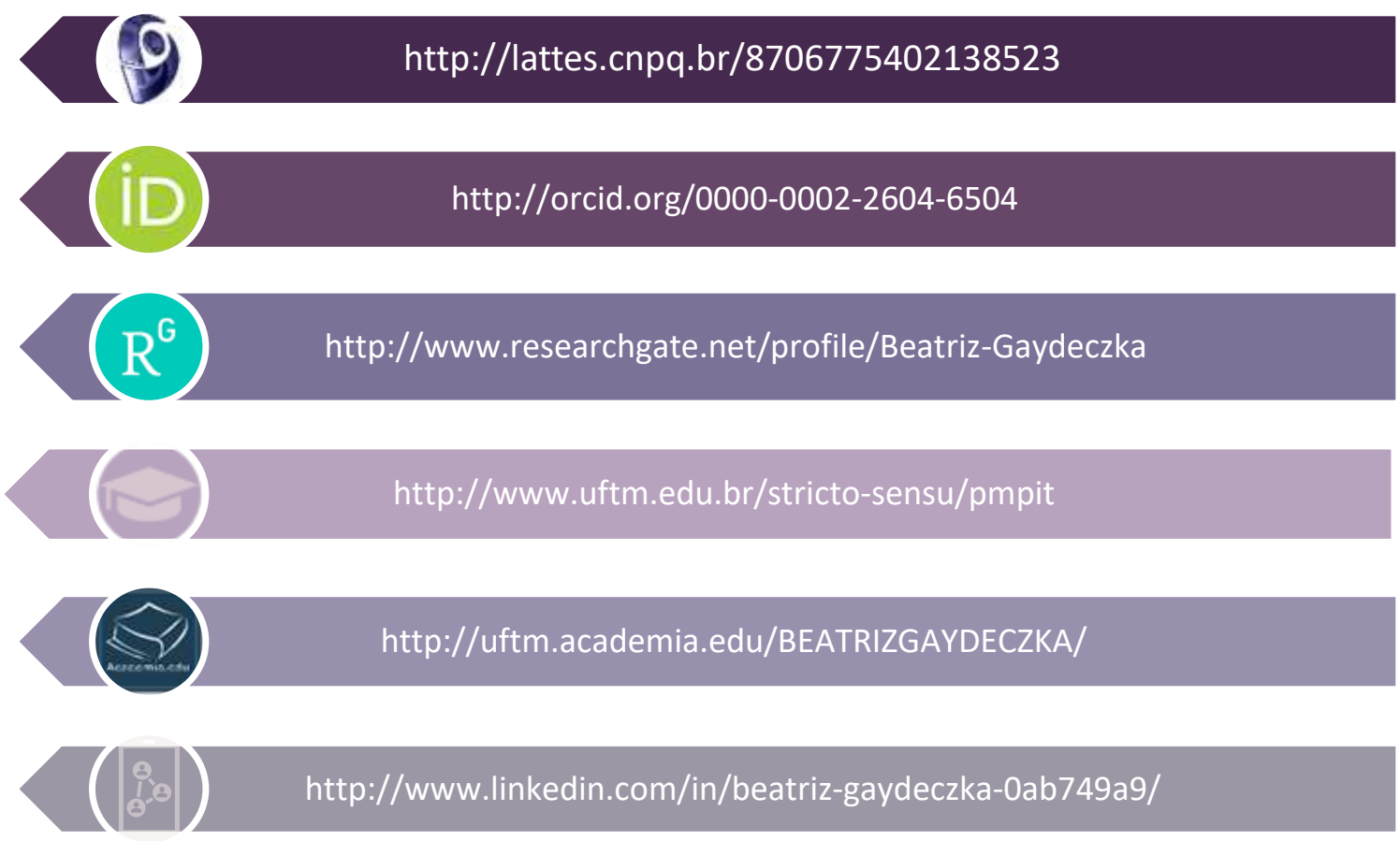

DOI: https://doi.org/10.32988/rep.v10n1.1118

Dossiê "Possibilidades de trabalho com a Língua Portuguesa"

v. 10

n. 1

$1-20$

e021002




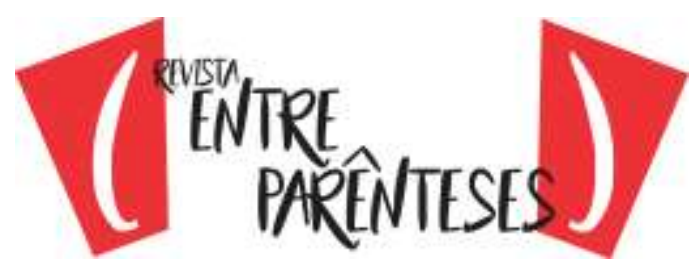

ISSN 2238-4502

\section{LEVANTAMENTO SOBRE HÁBITOS DE LEITURA E ESCRITA ENTRE ESTUDANTES DE ENGENHARIA}

\author{
Rafaela Giani de Resende ${ }^{1}$ \\ Universidade Federal do Triângulo Mineiro \\ (rafaelagiani@hotmail.com) \\ José Thiago da Rocha Neto \\ Universidade Federal do Triângulo Mineiro
}

\author{
Sofia Pelegrini Tristão \\ Universidade Federal do Triângulo Mineiro
}

\author{
Beatriz Gaydeczka² \\ Universidade Federal do Triangulo \\ Mineiro/FAPEMIG \\ (beatriz.gaydeczka@uftm.edu.br)
}

1 Introdução

É amplamente reconhecido que os profissionais da área de engenharia devem, fundamentalmente, possuir raciocínio lógico, habilidades com cálculos, domínio das ciências exatas e capacidade de solucionar problemas de forma calculada e precisa (BEER; McMURREY, 2009). No entanto, atualmente, o mercado de trabalho também exige do engenheiro competências relacionadas à comunicação eficaz, tanto no aspecto técnico (destreza digital), quanto na adequação ao contexto formal (capacidade de adaptabilidade) (SERAFIM, 2019).

As habilidades de comunicação ao longo dos últimos anos estão presentes entre as dez habilidades para o sucesso no mercado de trabalho, que são, segundo o Fórum Econômico Mundial (GRAY, 2016): solução de problemas complexos; pensamento crítico; criatividade; gestão de pessoas; coordenação com os outros; inteligência emocional; julgamento e tomada de decisões; orientação para serviço; negociação e flexibilidade cognitiva; comunicação. A maior parte da literatura separa as competências técnicas e profissionais (hards skills) das aptidões

\footnotetext{
1 Estudante de graduação em Engenharia do Instituto de Ciências Tecnológicas e Exatas da Universidade Federal do Triângulo Mineiro (UFTM).

${ }^{2}$ Graduação em Licenciatura em Língua Portuguesa (Unespar), Mestre em Linguística Aplicada (UNITAU), Doutora em Letras (USP), docente dos cursos de graduação em Engenharias e do Programa de Mestrado Profissional em Inovação Tecnológica do Instituto de Ciências Tecnológicas e Exatas da Universidade Federal do Triângulo Mineiro (UFTM).
}

Este artigo traz resultados da aplicação de uma metodologia de sequência didática desenvolvida no contexto do Projeto "Multiletramentos no ensino superior: leituras e produções textuais da cultura acadêmica", aprovado pela FAPEMIG sob no APQ - 01019-14.

DOI: https://doi.org/10.32988/rep.v10n1.1118

Dossiê "Possibilidades de trabalho com a Língua Portuguesa"

\begin{tabular}{|l|l|l|l|l|l|l|} 
Revista (Entre Parênteses) & Alfenas, MG & v. 10 & n. 1 & $1-20$ & e021002 & 2021 \\
\hline
\end{tabular}




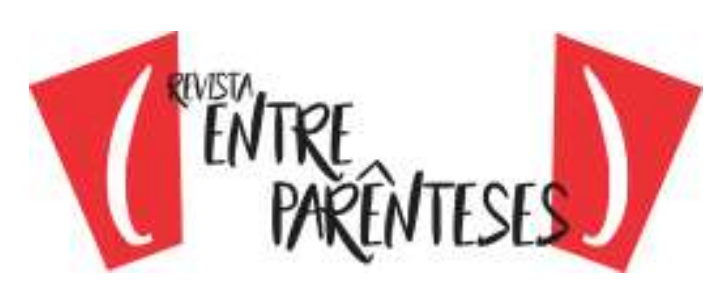

ISSN 2238-4502

pessoais (softs skills). As hards skills são as capacitações técnicas que um profissional pode comprovar por meio de diplomas, certificados de qualificação, testes práticos, cursos, entre outros. As softs skills são habilidades pessoais e intangíveis que um profissional possui. Estas não podem ser identificadas nem comprovadas por meio de certificações ou cursos e sim, pela convivência diária. 0 estado atual do debate considera esse argumento superado. Dessa forma, o desenvolvimento das chamadas softs skills pode e deve ser estimulado dentro das instituições de Ensino Superior (ROLIM; MACHADO-TAYLOR, 2006).

As novas Diretrizes Curriculares Nacionais para os cursos de Engenharia (DCNs) (BRASIL, 2019), no Capítulo II, definem o perfil e as competências esperadas pelo egresso. No Artigo 4. ${ }^{\circ}$, as DCNs apresentam as competências gerais que os cursos devem proporcionar ao longo da formação do engenheiro. Dessa forma, dentre as competências listadas, a relativa à dimensão comunicativa e linguística é:

V - comunicar-se eficazmente nas formas escrita, oral e gráfica:

a) ser capaz de expressar-se adequadamente, seja na língua pátria ou em idioma diferente do Português, inclusive por meio do uso consistente das tecnologias digitais de informação e comunicação (TDICs), mantendo-se sempre atualizado em termos de métodos e tecnologias disponíveis (BRASIL, 2019).

A competência $\mathrm{V}$ possui o foco no desenvolvimento de competências relacionadas à comunicação eficaz. Na engenharia a percepção ou a qualificação de produtos, de processos e até de pessoas como eficazes e eficientes é comum. Mas o que é ser eficiente/eficaz do ponto de vista da comunicação? Dentre as possibilidades destaca-se tornar comuns ideias, necessidades e projetos, persuadir (saber fazer uso da polidez) e gerar respostas (BLIKSTEIN, 1985). Além disso, a eficiência comunicativa está na adequação, ou seja, em ajustar a comunicação ao contexto e ao alcance de todos (destinatários, usuários), ter boa noção de adequação de vocabulário padrão e do tempo que se leva para produzir e enviar os textos. A eficiência de qualquer profissão - e com ela a sua utilidade - depende tanto da sua habilidade de fazer com que outras pessoas entendam seu trabalho quanto da própria qualidade do trabalho (DRUCKER, 1952 apud BARRASS, 1979).

Nessa perspectiva, as competências gerais apresentadas pelas DCN pressupõem o desenvolvimento de habilidades específicas, tais como as habilidades relacionadas à leitura (compreensão de sentidos) e à escrita (produção de textos). Na esfera profissional de engenheiros há necessidade não apenas de clareza e coerência a fim de que possam se expressar adequadamente em seu ofício, mas também de dispor de boa leitura e interpretação para compreender normas e projetos (FRANZEN; SCHLICHTING; HEINIG, 2011), reconhecendo

DOI: https://doi.org/10.32988/rep.v10n1.1118

Dossiê "Possibilidades de trabalho com a Língua Portuguesa"

\begin{tabular}{|l|l|l|l|l|l|l} 
Revista (Entre Parênteses) & Alfenas, MG & v. 10 & n. 1 & $1-20$ & e021002 & 2021 \\
\hline
\end{tabular}




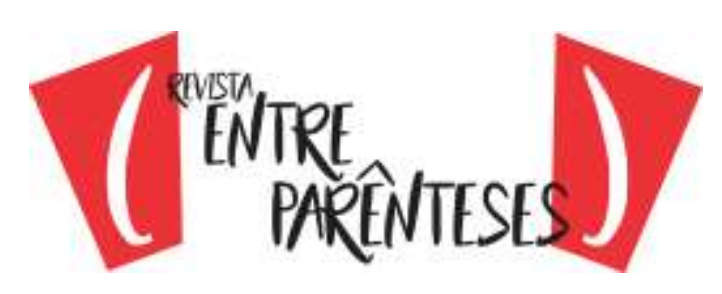

ISSN 2238-4502

nas normas e projetos as necessidades dos usuários e seus contextos ${ }^{3}$. Além da leitura, existe a necessidade pragmática de comunicar de forma oral, escrita e gráfica em muitas situações, seja em apresentações formais, em ligações de vendas ou no compartilhamento de ideias em uma reunião. Assim é preciso analisar a audiência (ouvintes e leitores) e gerenciar a ansiedade comunicativa nas diferentes modalidades linguísticas.

Como é papel da universidade trabalhar hards e softs skills de comunicação, é fundamental que os jovens profissionais ou cientistas demonstrem seus conhecimentos de forma clara, objetiva, coesa, confiante e de impacto. Nesse sentido, Barrass (1979) destaca, por exemplo, a importância da escrita acadêmica e explica como a defasagem de aprendizagem e a falta de valorização da escrita impacta de maneira ampla a vida:

Escrever é parte da ciência. Não obstante, muitos cientistas deixaram de receber treinamento na arte de escrever. Há uma certa ironia no fato de ensinarmos nossos cientistas e engenheiros a utilizarem instrumentos e técnicas, muitas dos quais jamais utilizarão em sua vida profissional, e, no entanto, não os ensinamos a escrever. Escrever é o que eles precisarão fazer todos os dias - como estudantes, como administradores, como executivos, como cientistas e engenheiros (BARRASS, 1979, p.4).

Diante do alerta de Barrass, é necessário que as práticas linguísticas utilizadas no mundo do trabalho ou acadêmico sejam desenvolvidas no Ensino Superior. Mas, apesar desse reconhecimento, a realidade nas universidades é a dominância de uma educação técnica e exata em detrimento do estudo da leitura e da escrita voltada para a atuação futura.

Além disso, observa-se em estudos como os de Barrass (1979), que parte dos engenheiros, cientistas e estudantes de engenharias são indiferentes à importância da escrita $\mathrm{e}$ da leitura em suas vidas, não se preocupam com essas competências e acreditam que elas não são importantes. Assim, como fazer para que a cultura da palavra escrita seja valorizada entre estudantes de engenharias?

Primeiramente, frente a esse desafio e questionamento, é necessário um ensino formal e sistêmico de gêneros textuais/discursivos na formação superior (MACHADO; LOUSADA; ABREU-TARDELLI, 2005), em segundo lugar, é necessário compreender o paradigma atual e local acerca do tema (BAZERMAN, 2006), pois nessa conjuntura estudos, tais como o presente, podem fornecer pistas acerca dos hábitos de leitura e escrita entre os jovens estudantes do Ensino Superior.

3 Usuários: aqueles com que o engenheiro interage, gerindo força de trabalho, liderando equipes multidisciplinares, tanto localmente quanto em rede.

DOI: https://doi.org/10.32988/rep.v10n1.1118

Dossiê "Possibilidades de trabalho com a Língua Portuguesa"

\begin{tabular}{|l|l|l|l|l|l|l} 
Revista (Entre Parênteses) & Alfenas, MG & v. 10 & n. 1 & $1-20$ & e021002 & 2021 \\
\hline
\end{tabular}




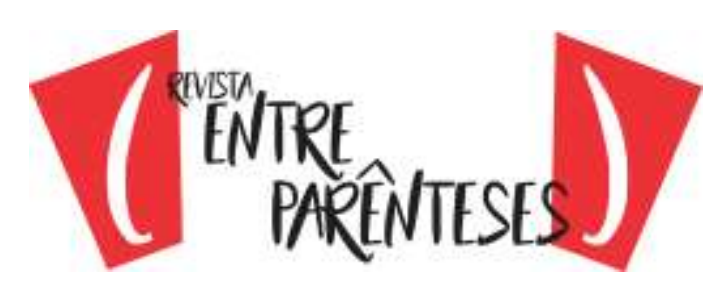

ISSN 2238-4502

Dessa forma, este artigo objetiva apresentar dados de um levantamento sobre os hábitos de leitura e escrita entre estudantes de engenharia, traçando o perfil e as expectativas quanto aos letramentos dos estudantes.

\section{Método de coleta de dados}

A motivação para este trabalho ocorreu em sala de aula na disciplina de Redação de Textos Técnicos e Científicos (RTTC) (no segundo semestre de 2019), durante a qual foi desenvolvida uma sequência didática para ensino-aprendizagem do gênero artigo científico, decorrente da aplicação de uma metodologia desenvolvida no contexto do Projeto "Multiletramentos no ensino superior: leituras e produções textuais da cultura acadêmica", aprovado pela FAPEMIG sob no APQ - 01019-14.

Para o desenvolvimento da coleta de dados foi realizado um levantamento junto aos estudantes de cursos integrais e presenciais das Engenharias: Ambiental, Civil, de Alimentos, de Produção, Elétrica, Mecânica e Química da Universidade Federal do Triângulo Mineiro, matriculados em duas turmas da disciplina RTTC, em um total de 55 alunos.

A aplicação do instrumento com tal temática "Hábitos de leitura e escrita" teve como base a realização de uma atividade didática proposta por Coscarelli e Mitre (2012): a) cada estudante responde a cinco perguntas; b) as respostas são agrupadas de acordo com suas semelhanças e frequência; c) é feita a organização de grupos por pergunta; d) cada grupo categoriza, discute e apresenta as respostas. Porém, essa atividade permitia a coleta de dados e discussão em um grupo restrito, limitando a comparação dos resultados com outras turmas. Em consequência disso, foi proposta a adaptação da atividade para um instrumento com perguntas objetivas.

Assim, o instrumento foi adaptado para um formulário no Google Forms disponível no link https://forms.gle/m94ZocaZmzrgoW1RA e enviado por e-mail aos estudantes entre os meses de setembro e outubro de 2019. A opção pela construção do instrumento nesse formato foi decorrente da necessidade de os estudantes compreenderem e aprenderem como é o processo de construção de um instrumento on-line de coleta de dados e, além disso, pudessem obter em uma única planilha os dados de diferentes turmas para análise e discussão.

Resumidamente o instrumento foi composto por sete questões, enfocando as seguintes temáticas: perguntas quantitativas - a existência ou não de hábitos de leitura e escrita; as dificuldades nos momentos de escrita; a percepção dos objetivos de uma disciplina de escrita nas engenharias; e perguntas qualitativas - a descrição dos materiais de leitura e escrita; a disposição para a melhoria de desempenho na escrita.

Quanto aos procedimentos de análise dos dados, aos dados quantitativos foi dado tratamento estatístico e aos dados qualitativos foi realizada análise de conteúdo. Dessa forma, as informações foram categorizadas, analisadas e generalizadas.

DOI: https://doi.org/10.32988/rep.v10n1.1118

Dossiê "Possibilidades de trabalho com a Língua Portuguesa"

\begin{tabular}{|l|l|l|l|l|l|l|}
\hline Revista (Entre Parênteses) & Alfenas, MG & v. 10 & n. 1 & $1-20$ & e021002 & 2021 \\
\hline
\end{tabular}




\section{("intrire}

ISSN 2238-4502

SANTOS, 2014). A esse respeito, Pinker (2016) afirma que as queixas sobre o declínio da língua são tão antigas quanto a invenção da tipografia. Hoje, segundo Pinker (2016), temos um corpo de pesquisas sobre a dinâmica mental da leitura: aumento e diminuição das cargas de memória à medida que os leitores entendem uma passagem, o incremento do conhecimento à medida que captam o sentido, os becos sem saída que podem desconsertar o leitor.

Tabela 1. Tipo e temática de gêneros citados pelos estudantes

\begin{tabular}{|c|c|c|}
\hline \multirow{2}{*}{ Temática } & Tipo de materiais de leitura & N. alunos \\
\hline \multirow{3}{*}{\begin{tabular}{c}
\multirow{2}{*}{$\begin{array}{c}\text { Interesse } \\
\text { pessoal }\end{array}$} \\
\cline { 2 - 3 }
\end{tabular}} & Literatura (romance, ficção, mitologia, crônicas) & 12 \\
\cline { 2 - 3 } & Livros de autoconhecimento e autoajuda & 2 \\
\cline { 2 - 3 } & Finanças e investimentos e empreendedorismo & 2 \\
\cline { 2 - 3 } & Religiosos & 1 \\
\hline \multirow{2}{*}{ Acadêmico } & Materiais acadêmicos (livros didáticos) & 11 \\
\cline { 2 - 3 } & Artigos científicos & 9 \\
\hline Redes Sociais & Redes sociais & 8 \\
\hline Informação & Jornais e revistas \\
\hline
\end{tabular}

Tendo em vista o aumento significativo da importância da relação entre linguagens e tecnologia, realizar a leitura e a escrita de textos de forma satisfatória torna-se cada vez mais importante para estudantes de cursos como os de Engenharia. Entretanto, a compreensão de textos é o ponto fraco do estudante brasileiro, devido ao desleixo com o assunto no Ensino Médio, problema que se torna indisfarçável na faculdade com a necessidade de leituras mais complexas e longas (GUIMARÃES, 2012, p. 109).

Considerando a temática do hábito de escrita (Figura 2), observa-se que 59\% dos estudantes afirmam que possuem o hábito de escrita. No entanto, em comparação com os dados da Figura 1, nota-se que os estudantes afirmam escrever menos do que leem.

Esse resultado contribui para o retrógrado pensamento de que engenheiros não sabem escrever (FRANZEN; SCHLICHTING; HEINIG, 2013). No entanto, segundo Vieira e Faraco (2019a), a realidade demonstra o fato de que a escrita é uma prática que exige esforços e conhecimentos. Devido a isso, ela é mais complexa que a leitura em todos os cursos universitários. Portanto, especificamente, são necessários maior atenção e maior incentivo à escrita dos futuros engenheiros.

DOI: https://doi.org/10.32988/rep.v10n1.1118

Dossiê "Possibilidades de trabalho com a Língua Portuguesa"

\begin{tabular}{|l|l|l|l|l|l|l|} 
Revista (Entre Parênteses) & Alfenas, MG & v. 10 & n. 1 & $1-20$ & e021002 & 2021 \\
\hline
\end{tabular}




\section{(")}

ISSN 2238-4502

Figura 2. Hábitos de escrita entre estudantes de Engenharia

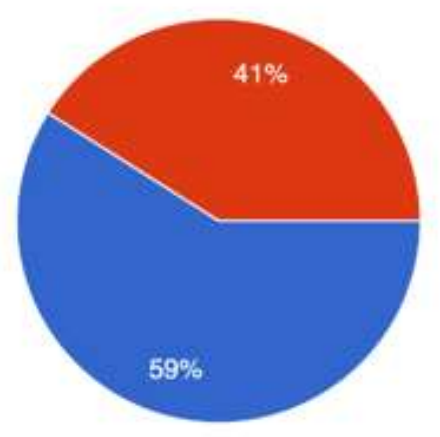

$$
\begin{aligned}
& \text { Sim } \\
& \text { Năo }
\end{aligned}
$$

Em relação aos itens apresentados na Tabela 2, os estudantes tinham a opção de apresentar até três materiais ou gêneros de textos que mais frequentemente utilizam. Dessa forma, 87\% dos estudantes afirmam escrever gêneros relacionados à esfera acadêmica; dessa parcela, 50\% escrevem exclusivamente para cumprir às exigências dos cursos de formação. Além do mais, 43\% afirmam escrever nas redes sociais a fim de dar opiniões, criticar, elogiar e divulgar postagens.

Tabela 2. Gêneros textuais/discursivos escritos pelos estudantes

\begin{tabular}{|c|l|c|}
\hline \multicolumn{1}{|c|}{ Esfera } & Gêneros textuais/discursivos ou ambientes & $\begin{array}{c}\text { N. de } \\
\text { alunos }\end{array}$ \\
\hline \multirow{4}{*}{ Acadêmica } & Relatórios acadêmicos & 10 \\
\cline { 2 - 3 } & Apenas faculdade, trabalhos & 6 \\
\cline { 2 - 3 } & Trabalho de conclusão de curso, artigos e IC & 5 \\
\cline { 2 - 3 } & Resumos & 5 \\
\hline \multirow{3}{*}{ Redes sociais } & Redes sociais & 10 \\
\cline { 2 - 3 } & Textos de post para divulgação & 1 \\
\hline \multirow{3}{*}{ Profissional } & Ambiente de trabalho & 3 \\
\cline { 2 - 3 } & Cartas ou e-mails & 1 \\
\cline { 2 - 3 } & Atas & 1 \\
\hline
\end{tabular}

Fazendo uma análise comparativa entre a Tabela 1 e a Tabela 2, pode-se observar que há diversidade de materiais quando se trata de leitura, mas, quando se refere à escrita, a

DOI: https://doi.org/10.32988/rep.v10n1.1118

Dossiê "Possibilidades de trabalho com a Língua Portuguesa"

\begin{tabular}{|l|l|l|l|l|l|l|} 
Revista (Entre Parênteses) & Alfenas, MG & v. 10 & n. 1 & $1-20$ & e021002 & 2021 \\
\hline
\end{tabular}




\section{(")}

ISSN 2238-4502

variedade de materiais fica restrita às esferas acadêmica, mídia digital e profissional. "Isso de deve, em parte, talvez, ao fato de que normalmente falamos e escutamos mais do que lemos, e lemos mais do que escrevemos" (VIEIRA; FARACO, 2019b, p. 7).

Ao se acessar as redes sociais, observa-se que muitos usuários da internet valorizam a linguagem clara, gramatical, em boa ortografia e pontuação, não só nos livros impressos e nas mídias tradicionais, mas também nos zines eletrônicos, nos blogs, nos verbetes da Wikipédia, nas avaliações de consumidores, e mesmo em boa parte dos e-mails. Levantamentos mostraram que universitários estão escrevendo mais do que gerações anteriores, e que não fazem um número maior de erros por página (LUNSFORD, 2013 apud PINKER, 2016).

Quanto às dificuldades enfrentadas durante o processo de escrita, as mencionadas em maior número são "colocar e organizar as ideias no papel" e "dominar as regras gramaticais" (Figura 3). Segundo Franzen e Heinig (2012), engenheiros, formados e atuantes em sua área, apontam dificuldades em colocar as ideias no papel de modo claro e objetivo e em abordar assuntos técnicos com uma linguagem clara e de fácil compreensão.

Esse problema, em alguns casos, é chamado "maldição do conhecimento". Ou seja, incapacidade de explicar de modo acessível algo complexo. A clareza e a objetividade pressupõem a capacidade de avaliar o grau de conhecimento e de habilidades das outras pessoas, a fim de que o leitor saiba do que está falando, compreenda as fases de uma cena, perceba os pormenores necessários à compreensão (PINKER, 2016).

Cabe também observar que os tópicos "colocar as ideias no papel”, "não ter assunto para dizer" são aspectos que têm relação direta com a leitura. Não se trata de ser prolixo ou preencher o texto com blá, blá, blá, pois a alimentação temática dos textos depende do repertório e da densidade informativa. Quem tem proficiência de leitura sabe observar a forma, o estilo, o modo de dizer dos textos e dos autores lidos e reconhecer tendências e movimentos retóricos dos gêneros. Além disso, "por mais aulas que você tenha, palestras que presencie, discussões de que participe, a leitura de textos acadêmicos escritos é indispensável para a compreensão, seleção e organização de informações e pontos de vista consistentes e diversificados. Em suma: sem leitura na universidade, não haverá escrita na universidade." (VIEIRA; FARACO, 2019b, p. 23, grifo dos autores). Ou seja, existe a necessidade de que um universitário conheça diferentes discursos que circulam socialmente sobre um assunto - e isso deve ser feito por meio da leitura de bons textos sobre o tema - assim se dá a preparação para a compreensão e escrita de algo sobre o assunto.

DOI: https://doi.org/10.32988/rep.v10n1.1118

Dossiê "Possibilidades de trabalho com a Língua Portuguesa"

\begin{tabular}{|l|l|l|l} 
Revista (Entre Parênteses) & Alfenas, MG & v. 10 & n. 1 \\
\hline
\end{tabular}




\section{(")}

ISSN 2238-4502

Tabela 3. 0 que é necessário para melhorar a escrita

\begin{tabular}{|c|c|}
\hline Esforço pessoal & N. de alunos \\
\hline Ler mais & 22 \\
\hline Escrever mais & 20 \\
\hline Dedicação e esforço & 5 \\
\hline Aprimoramento linguístico, vocabulário e pensamento crítico & 3 \\
\hline
\end{tabular}

De fato, construir uma cultura que valorize a prática letrada é um dos fatores mais impactantes para que alguém seja capaz de realizar uma boa escrita. A escrita e a leitura, realizadas com frequência, auxiliam para um aumento significativo do vocabulário e do conhecimento de princípios estruturais, textuais, discursivos e gramaticais. Nesse sentido, é possível ver reflexos da opinião dos estudantes a partir dos pensamentos de Vieira e Faraco (2019a), os quais defendem que a leitura possui a função de aprimorar o domínio da escrita já que aquela permite a expansão de repertório e de exemplos para a prática desta. Ademais, apesar de que "bons escritores são sempre leitores ávidos" (VIEIRA; FARAC0, 2019a, p.43), a leitura sozinha não basta para escrever bem, é preciso praticar a escrita.

\section{Conclusão}

Ao realizar este levantamento, buscou-se compreender aspectos relacionados aos hábitos de leitura e escrita entre um grupo de estudantes de engenharia (matriculados em uma disciplina de redação de textos técnicos e científicos) e destacar a percepção desses a respeito da importância desses conhecimentos para a vida acadêmica e profissional.

O estudo feito mostrou que uma parcela significativa dos participantes da pesquisa não possui esses hábitos incorporados na vida. A partir dos resultados obtidos, nota-se que uma porcentagem significativa dos estudantes não possui hábitos de leitura e escrita. Aqueles que os possuem relacionam esses hábitos às atividades da esfera acadêmica. De maneira geral, os dados mostraram que os estudantes estão exercendo essas habilidades principalmente nas redes sociais, mas desconsideram a leitura e a escrita realizadas nas redes sociais como práticas válidas para o desenvolvimento de tais habilidades. Essa desconsideração pode ser relacionada a concepção de que os hábitos de leitura e escrita são aplicados apenas para textos formais, "físicos", "extensos" e com a ideia de que essas práticas, via redes sociais, são utilizadas somente para entretenimento.

DOI: https://doi.org/10.32988/rep.v10n1.1118

Dossiê "Possibilidades de trabalho com a Língua Portuguesa"

\begin{tabular}{|l|c|c|c|c|c|c|}
\hline Revista (Entre Parênteses) & Alfenas, MG & v. 10 & n. 1 & $1-20$ & e021002 & 2021 \\
\hline
\end{tabular}


Em relação às dificuldades apontadas durante a escrita e aos possíveis esforços empreendidos para a melhoria do desempenho da escrita, os resultados mostram a ligação direta entre ambos.

Quanto ao tipo de conhecimento que uma disciplina de redação e escrita pode proporcionar, muitos estudantes relacionam esse aprendizado com o mundo profissional, ao esperarem aprender técnicas para se desenvolverem pessoal e profissionalmente. Portanto, é perceptível a preocupação dos estudantes e a necessidade da boa escrita para o futuro profissional.

Nas discussões dos resultados, pesquisas e materiais correlatos como Franzen; Heinig (2012), Ribeiro; Guimarães; Silva (2012) Vieira; Faraco (2019a e b) complementam-se por meio da identificação de problemas similares e de propostas para a sua solução. Espera-se que essa análise contribua para o incentivo à leitura e à escrita e que docentes e discentes possam discutir ações concretas para aprimorar a prática desses hábitos.

Referências

BARRASS, R. Cientistas precisam escrever: guia de redação para cientistas, engenheiros e estudantes. São Paulo: EDUSP, 1979.

BAZERMAN, C. O que é interessante? In: BAZERMAN, C.; HOFFNAGEL, J. C.; DIONÍSIO, A. P. (org.). Gênero, agência e escrita. São Paulo: Cortez, 2006.

BEER, D.; McMURREY, D. A guide to writing as an engineer. 3. ed. Danvers, MA: Wiley, 2009. BLIKSTEIN, I. Técnicas de comunicação escrita. São Paulo: Ática, 1985.

BORK, A. V. et al. Mapeamento das iniciativas de escrita em língua materna na educação superior: resultados preliminares. Revista Prolíngua, v. 9, n. 1, p. 2-14, 2014. Disponível em: https://bazerman.education.ucsb.edu/sites/default/files/docs/Bazerman2014ARTetalProlin guaMapeamento.pdf. Acesso em: 29 dez. 2020.

BRASIL. Resolução n. 2, de 24 de abril de 2019. Institui as diretrizes curriculares nacionais do curso de graduação em Engenharia. Brasília: DOU, 26 de abril de 2019, Seção 1, p. 43-44. Disponível em:

http://portal.mec.gov.br/index.php?option=com_docman\&view=download\&alias=112681rces002-19\&category_slug=abril-2019-pdf\&Itemid=30192. Acesso em 11 mai. 2020.

COSCARELLI, C. V.; MITRE, D. Oficina de leitura e produção de textos: livro do professor. 1. reimp. Belo Horizonte: Editora UFMG, 2012.

FISCHER, A.; COLAÇO, S. F. Práticas de escrita em Ciências Biológicas: letramentos acadêmicos (re)contextualizados. Raído, v. 11, n. 27, p. 438, 18 ago. 2017. DOI:

DOI: https://doi.org/10.32988/rep.v10n1.1118

Dossiê "Possibilidades de trabalho com a Língua Portuguesa"

\begin{tabular}{|l|l|l|l|l|l|l} 
Revista (Entre Parênteses) & Alfenas, MG & v. 10 & n. 1 & $1-20$ & e021002 & 2021 \\
\hline
\end{tabular}




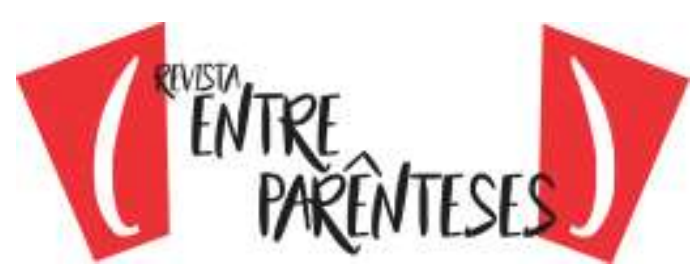

ISSN 2238-4502

https://doi.org/10.30612/raido.v11i27.6751. Disponível em: https://ojs.ufgd.edu.br/index.php/Raido/article/view/6751. Acesso em: 29 dez. 2020.

FRANZEN, B. A; HEINIG, O. L. de O. M. Letramentos situados: a linguagem no campo de trabalho de engenheiros. Atos de Pesquisa em Educação, 2012. Disponível em:

https://bu.furb.br/ojs/index.php/atosdepesquisa/article/view/3466. Acesso em: 29 dez. 2020.

FRANZEN, B. A.; SCHLICHTING, T. D. S.; HEINIG, O. L. O. M. A leitura e a escrita no mundo do trabalho: o que dizem os engenheiros. In: Anais do 39o Congresso Brasileiro de Educação em Engenharia de Produção, 2011. Disponível em:

http://www.abenge.org.br/cobenge/arquivos/8/sessoestec/art1772.pdf. Acesso em: 29 dez. 2020.

FRANZEN, B. A.; SCHLICHTING, T. S.; HEINIG, O. L. O. M. Engenheiros: uma construção de múltiplas identidades. In: XLI Congresso Brasileiro de Educação em Engenharia. Gramado, 2013. Disponível em: https://www.fadep.br/engenhariaeletrica/congresso/pdf/117101 1.pdf. Acesso em: 29 dez. 2020.

GRAY, A. Top 10 skills you need to thrive in the fourth industrial revolution. World Economic Forum. 19 jan. 2016. Disponível em:

https://www.weforum.org/agenda/2016/01/the-10-skills-you-need-to-thrive-in-the-fourthindustrial-revolution/. Acesso em: 29 dez. 2020.

GUIMARÃES, T. C. Comunicação e linguagem. São Paulo: Pearson, 2012.

MACHADO, A. R.; LOUSADA, E.; ABREU-TARDELLI, L. S. Planejar gêneros acadêmicos. São Paulo: Parábola, 2005.

MOTTA-ROTH, D.; HENDGES, G. R. Produção textual na universidade. São Paulo: Parábola Editorial, 2010.

RIBEIRO, A. E.; GUIMARÃES, F.I.; COSTA DA SILVA, e. S. Aulas de Português na formação de engenheiros: expectativas e concepções de alunos e professores de instituição pública em Minas Gerais. Scripta, v. 16, n. 30, p. 117-136, 17 jul. 2012. Disponível em:

http://periodicos.pucminas.br/index.php/scripta/article/view/4243. Acesso em: 29 dez. 2020.

ROLIM, C.; MACHADO-TAYLOR, M. L. Hard X Soft Skills e Desemprego entre Graduados Universitários. 2016. Fórum da Gestão do Ensino Superior nos Países e Regiões de Língua Portuguesa, Ilhéus, 2016. Disponível em:

http://www.aforges.org/wp-content/uploads/2016/11/1-Cassio-Romim-Maria-de-LourdesTaylor -Hard-X-soft-skills.pdf. Acesso em: 29 dez. 2020.

SANTOS, V. L. C.; SANTOS, J. E. As redes sociais digitais e sua influência na sociedade e educação contemporâneas. HOLOS, v. 6, 2014, pp. 307-328. DOI:

DOI: https://doi.org/10.32988/rep.v10n1.1118

Dossiê "Possibilidades de trabalho com a Língua Portuguesa"

\begin{tabular}{|l|c|c|c|c|c|c|} 
Revista (Entre Parênteses) & Alfenas, MG & v. 10 & n. 1 & $1-20$ & e021002 & 2021 \\
\hline
\end{tabular}




\section{(")}

ISSN 2238-4502

https://doi.org/10.15628/holos.2014.1936 Disponível em: https://www.redalyc.org/pdf/4815/481547175023.pdf. Acesso em: 29 dez. 2020.

SERAFIM, M. P. Como será o cenário da Educação Superior em 2030? Avaliação: Revista da Avaliação da Educação Superior (Campinas), v. 24, n. 3, p. 569-572, 2019. DOI: https://doi.org/10.1590/s1414-40772019000300001 Disponível em: http://www.scielo.br/scielo.php?script=sci_arttext\&pid=S141440772019000300569\&lng=pt\&nrm=iso\&tlng=pt . Acesso em: 29 dez. 2020.

VIEIRA, F. E.; FARACO, C. A. Escrever na universidade: fundamentos. São Paulo: Parábola, 2019a.

Escrever na universidade: texto e discurso. 1. ed. São Paulo: Parábola, 2019b.

ZANOTELLO, M.; ALMEIDA, M. J. P. de. Leitura de um texto de divulgação científica em uma disciplina de física básica na educação superior. Ensaio Pesquisa em Educação em Ciências, v. 15, n. 3, set-dez, 2013, pp. 113-130. DOI: https://doi.org/10.1590/1983-21172013150307. Disponível em: https://www.scielo.br/scielo.php?script=sci_arttext\&pid=S198321172013000300113\&lng=en\&nrm=iso\&tlng=pt. Acesso em: 29 dez. 2020.

Recebido em 28/03/2020

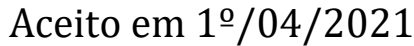

Publicado em 30/06/2021

DOI: https://doi.org/10.32988/rep.v10n1.1118

Dossiê "Possibilidades de trabalho com a Língua Portuguesa"

n. 1

$1-20$




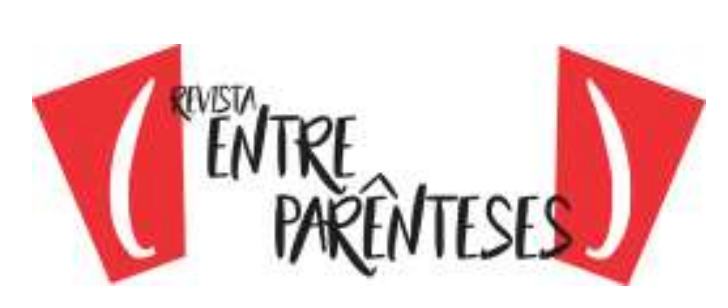

ISSN 2238-4502

\title{
SURVEY ABOUT READING AND WRITING HABITS AMONG ENGINEERING STUDENTS
}

\author{
Rafaela Giani de Resende \\ Universidade Federal do Triângulo Mineiro \\ (rafaelagiani@hotmail.com) \\ José Thiago da Rocha Neto \\ Universidade Federal do Triângulo Mineiro
}

\author{
Sofia Pelegrini Tristão \\ Universidade Federal do Triângulo Mineiro
}

\author{
Beatriz Gaydeczka \\ Universidade Federal do Triangulo \\ Mineiro/FAPEMIG \\ (beatriz.gaydeczka@uftm.edu.br)
}

\begin{abstract}
This paper, the product of the experience of a didactic sequence for learning academic genres, aims to present data from a survey on reading and writing habits among engineering students. An online tool (Google Forms) was applied with five questions (open and closed) on the topic. It was found that most of the students have a reading habit, with the majority dedicating themselves to reading academic articles and books. Regarding the habit of writing, it was observed that it occurs less frequently and is linked to the needs of the course, highlighting the greatest difficulties: a) transferring ideas to paper; $b$ ) organize ideas in a coherent way. In general, the existence of disciplines that focus on specific writing for engineering students, together with the student's own effort, would help the use of techniques to develop writing both personally and professionally, such as the production of technical texts, instruction manuals or operational standards.
\end{abstract}

Keywords: Skills. Communication skills. Textual / discursive genres.

DOI: https://doi.org/10.32988/rep.v10n1.1118

Dossiê "Possibilidades de trabalho com a Língua Portuguesa"

\begin{tabular}{|c|c|c|c|c|c|}
\hline Revista (Entre Parênteses) & Alfenas, MG & v. 10 & n. 1 & $1-20$ & $\mathrm{e} 021002$ \\
\hline $\begin{array}{r}\text { Depart } \\
\text { Rua Gabriel } \\
\text { https://publicaco }\end{array}$ & $\begin{array}{l}\text { Universid } \\
\text { nto de Letras - } \\
\text { teiro da Silva, } 7 \\
\text { nifal-mg.edu.l }\end{array}$ & $\begin{array}{l}\text { ederal } \\
\text { uto de } \\
\text { Alfenas } \\
\text { vistas }\end{array}$ & $\begin{array}{l}\text { nas } \\
\text { s Hu } \\
\text { CEP } \\
\text { php }\end{array}$ & $\begin{array}{l}\text { e Letr } \\
1-001 \\
\text { epare }\end{array}$ & asil \\
\hline
\end{tabular}




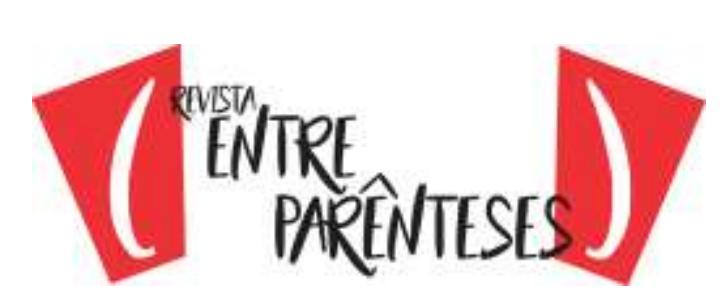

ISSN 2238-4502

\title{
ENCUESTA SOBRE ESCRITURA Y HABITOS DE ESCRITURA ENTRE ESTUDIANTES DE INGENIERÍA
}

\author{
Rafaela Giani de Resende \\ Universidade Federal do Triângulo Mineiro \\ (rafaelagiani@hotmail.com) \\ José Thiago da Rocha Neto \\ Universidade Federal do Triângulo Mineiro
}

\author{
Sofia Pelegrini Tristão \\ Universidade Federal do Triângulo Mineiro
}

Beatriz Gaydeczka

Universidade Federal do Triangulo

Mineiro/FAPEMIG

(beatriz.gaydeczka@uftm.edu.br)

\begin{abstract}
Resumen
Este documento, producto de la experiencia de una secuencia didáctica para aprender géneros académicos, tiene como objetivo presentar datos de una encuesta sobre hábitos de lectura y escritura entre estudiantes de ingeniería. Se aplicó una herramienta en línea (Formularios de Google) con cinco preguntas (abiertas y cerradas) sobre el tema. Se descubrió que la mayoría de los estudiantes tienen un hábito de lectura, y la mayoría se dedica a leer artículos y libros académicos. Con respecto al hábito de escribir, se observó que ocurre con menos frecuencia y está relacionado con las necesidades del curso, destacando las mayores dificultades: a) transferir ideas al papel; b) organizar ideas de manera coherente. En general, la existencia de disciplinas que se centran en la escritura específica para estudiantes de ingeniería, junto con el propio esfuerzo del estudiante, ayudaría al uso de técnicas para desarrollar la escritura tanto personal como profesionalmente, como la producción de textos técnicos, manuales de instrucción o estándares operativos.
\end{abstract}

Palabras clave: Habilidades. Habilidades de comunicación. Géneros textuales / discursivos.

DOI: https://doi.org/10.32988/rep.v10n1.1118

Dossiê "Possibilidades de trabalho com a Língua Portuguesa"

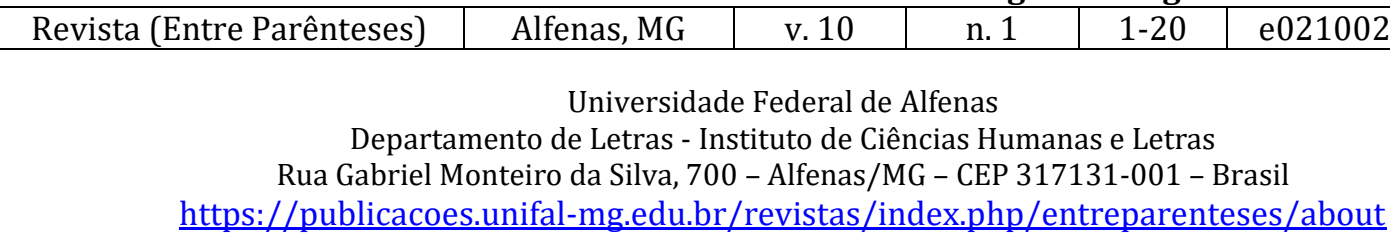

